Paul H. Mayo

Eric Maury

\section{Echography is mandatory for the initial management of critically ill patients: We are not sure}

Received: 5 August 2014

Accepted: 18 August 2014

Published online: 7 October 2014

(C) Springer-Verlag Berlin Heidelberg and ESICM 2014

For contrasting viewpoints please see doi:

10.1007/s00134-014-3465-7 and doi:10.1007/s00134-014-3469-3.

P. H. Mayo (৫)

Division of Pulmonary, Critical Care, and Sleep Medicine, North Shore/LIJ Medical Center, Hofstra North Shore/LIJ School of Medicine, Hempstead, NY, USA

e-mail: mayosono@gmail.com

\section{E. Maury}

Service de Reanimation Medicale, Hopital Saint-Antoine,

Assistance Publique- Hopitaux de Paris, 184 rue du Faubourg

Saint-Antoine, Paris 75571, France

e-mail: eric.maury@sat.aphp.fr

We have been assigned the "we do not know" position regarding the statement "Echography is mandatory for the initial management of the critically ill patient."

We understand echography to be synonymous with critical care ultrasonography (CCUS) which includes thoracic, abdominal, vascular, and cardiac ultrasonography [1]. Implicit in the definition of CCUS is that image acquisition and interpretation are performed at the point of care by the frontline intensivist. There is no doubt that certain applications of CCUS, such as renal ultrasonography for evaluation of kidney failure [2], pleural ultrasonography for identification and characterization of pleural fluid [3], ultrasonography for diagnosis of deep venous thrombosis [4], and ultrasonography for procedural guidance, should be routine in the intensive care unit. For the latter application, ultrasonography for guidance of vascular access should be considered as mandatory [5], while the use of ultrasonography in the patient on ventilatory support makes thoracentesis both feasible and safe [6].

However, the statement as written focuses attention on the initial management of the critically ill patient, i.e. the patient who presents with life-threatening cardiopulmonary failure. Our discussion will concentrate on whether intensivist performed ultrasonography is mandatory for the initial management. This would typically include a whole body approach that combines elements of cardiac, thoracic, vascular and limited abdominal ultrasonography as described initially by Lichtenstein et al. and more recently by Volpicelli et al. [7, 8]. We adopt a position of clinical equipoise for the following reasons.

1. There is no study that shows improvement in patient outcome with early use of point of care ultrasonography (e.g., cardiac, thoracic, abdominal, and vascular diagnostic) for the management of the patient with cardiopulmonary failure. On the other hand, echography has been demonstrated to be useful in identifying the cause for the cardiopulmonary failure. This is presumed to lead to management that will improve patient outcome, but compared to what? Is it truly known that early echography is superior to the traditional tools of the trade: the history, physical examination, standard radiography, and initial laboratory evaluation combined with clinical judgment and sound physiological reasoning? While the ultrasonography images are magical and the technology is seductive, does it actually improve patient outcome? We are waiting for more articles on this topic, because the literature does not currently demonstrate outcome improvement.

2. One means of determining whether ultrasonography actually improves patient outcome is to subject it to a randomized controlled trial (RCT). Proponents of ultrasonography will question why the modality is subject to such scrutiny, when other imaging methods 
such as radiographs and computed tomography are not. They will also point out that many aspects of critical care practice are not based upon any RCT, including the use of ventilators, invasive vascular access, continuous monitoring of vital signs, or even the physical examination. Other impediments can be envisioned. Critical care teams that utilize ultrasonography will be loath to join such a RCT, meaningful outcome variables will be difficult to identify and measure, and the complexity of the patient population presents major challenges in terms of achieving a wellbalanced study. It is possible that ultrasonography will be widely accepted based simply upon its intuitively obvious utility.

3. To describe echography as mandatory implies that a clinician at the bedside of the critically ill patient will perform the scan. This requires that the frontline intensivist be highly competent in critical care ultrasonography. What proportion of intensivists possess this skill level? In North America, very few intensivists have definitive skill in critical care ultrasonography. The same holds in Europe and the Asia Pacific region. The experts who insist on mandatory echography need only to observe the big national courses in the USA and at the ESICM annual meeting to witness hundreds of well-intentioned and motivated intensivists who are just starting their training sequence, and who then return to their home hospitals with limited skill level. It will be years before a generation of intensivists have true mastery of CCUS. We fear the combination of mandatory echography with incompetence in echography. This also raises the possibility that the intensivist who is actually competent in CCUS will become overconfident in their skill. Competence requires an understanding of the limits of competence. The radiologist and cardiologist have expert level knowledge that is not in the domain of CCUS, and not every clinical question can be answered at the bedside with CCUS. We also fear the intensivist with too much confidence in their own skill.

4. This problem of mandating the use of echography without consideration of the issue of competence has a flip side to it. The literature that demonstrates the utility of ultrasonography for the rapid diagnosis of cardiopulmonary failure is generally published by intensivists who are highly skilled in its application. They have reached a point of expertise and commitment where echography should be mandatory in their intensive care unit (ICU) or emergency department, but not necessarily in every ICU. We hope that they would start to include in their reports patients with multiple diagnoses, as this group of patients has generally been systematically excluded from the studies showing the utility of ultrasonography for diagnosis of cardiopulmonary failure. We need evidence of the utility of ultrasonography in real world settings where patients frequently have more than one cause of cardiopulmonary failure, and where the intensivist has a lesser skill level than the expert scanner.

5. We both have highly competent, productive, enthusiastic, and reliable colleagues who are heavyhitting frontline intensivists and who do not perform point of care ultrasonography in the ICU. The stipulation that echography is mandatory calls into question their competence as bedside clinicians. This is both wrong and offensive, given their excellent function in the ICU, which we see on a daily basis. It implies that the intensivist that does not use ultrasonography is somehow negligent. An intensivist can deliver superb care without having knowledge of bedside ultrasonography. Capability in echography is not a requirement but a recommended choice for evaluation of cardiopulmonary failure.

6. The widespread use of point of care ultrasonography may degrade other important aspects of critical care medicine, such as classic hemodynamic analysis, respiratory physiology, chest radiograph interpretation, physical examination, and the all-important history. The ultrasonography images are impressive, the newness of the field is exciting, and capable faculty are convincing advocates for mandatory echography on all critically ill patients in the ICU.

Table 1 Echography is mandatory for the initial management of critically ill patients

Summary of the "we are not sure" position

1. There is no literature that demonstrates that critical care ultrasonography alters patient outcome

2. It is not likely that a randomized control trial addressing the question will ever be performed

3. The mandatory use of ultrasonography requires that the intensivist have a high level of competence in the performance of critical care ultrasonography. The majority of intensivists do not have this skill level

4. The literature that supports the utility of critical care ultrasonography for the diagnosis of cardiopulmonary failure is presented by expert level scanners and generally excludes patients with multiple diagnoses. Most intensivists are not expert level, and patients often have multiple confounding diagnoses

5. Many highly effective frontline intensivists do not use ultrasonography, and instead use well-established alternative methods to diagnose cardiopulmonary failure to good effect

6. Untoward emphasis on ultrasonography may have the effect of degrading training and skill at other important aspects of critical care including mastery of the physical examination of the patient with cardiopulmonary failure 
However, we are concerned that the dominance of ultrasonography will displace training time required for other important aspects of critical care medicine. We particularly highlight our concern that the widespread use of ultrasonography may degrade the importance of the history and physical examination in the urgent evaluation of the patient with cardiopulmonary failure. Ultrasonography is an extension of the physical examination, but not its replacement. It should always be combined with the time honored skills of visual assessment, palpation, auscultation, and, on occasion, olfaction. Unlike the radiologist or consultative cardiologist, the intensivist deploys all aspects of the physical examination in conjunction with point of care ultrasonography in order to integrate the results into a rational diagnostic and management strategy.
All this does not discount the utility of point of care ultrasonography. We propose a modification of the statement as follows:

"Echography is useful for the initial management of the critically ill patient; however, it must be performed by a clinician who is fully competent in image acquisition, image interpretation, and the cognitive base of the field; and who is aware of the limitations of CCUS. Otherwise, the clinician should rely on other highly effective alternative means for the diagnosis of cardiopulmonary failure. While no clinician should feel that echography is mandatory in the ICU, it does have utility for rapid diagnosis of cardiopulmonary failure. We encourage, but do not mandate its use." (see Table 1).

Conflicts of interest The authors have no conflict of interest to declare related to the subject of this manuscript.

\section{References}

1. Mayo PH, Beaulieu Y, Doelken P, Feller-Kopman D, Harrod C, Kaplan A, Oropello J, Vieillard-Baron A, Axler O, Lichtenstein D, Maury E, Slama M, Vignon P (2009) American College of Chest Physicians/La Société de Réanimation de Langue Française statement on competence in critical care ultrasonography. Chest 135:1050-1060

2. Chalumeau-Lemoine L, Baudel JL, Das V, Arrivé L, Noblinski B, Guidet B, Offenstadt G, Maury E (2009) Results of short-term training of naïve physicians in focused general ultrasonography in an intensive-care unit. Intensive Care Med 35:1767-1771

3. Volpicelli G, Elbarbary M, Blaivas M, Lichtenstein DA, Mathis G, Kirkpatrick AW, Melniker L, Gargani L, Noble VE, Via G, Dean A, Tsung JW, Soldati G, Copetti R, Bouhemad B, Reissig A, Agricola E, Rouby JJ, Arbelot C, Liteplo A, Sargsyan A, Silva F, Hoppmann R, Breitkreutz R, Seibel A, Neri L, Storti E, Petrovic T, International Liaison Committee on Lung Ultrasound (ILCLUS) for International Consensus Conference on Lung Ultrasound (ICCLUS) (2012) International evidencebased recommendations for point-of-care lung ultrasound. Intensive Care Med 38:577-591
4. Kory PD, Pellecchia CM, Shiloh A, Mayo PH, Koenig S (2011) Accuracy of ultrasonography performed by critical care physicians for the diagnosis of deep venous thrombosis. Chest 139:538-542

5. Lamperti M, Bodenham AR, Pittiruti M, Blaivas M, Augoustides JG (2012) Internation evidence-based recommendation on ultrasound-guided vascular access. Intesive Care Med 38:105-1117

6. Lichtenstein D, Hulot JS, Rabiller A et al (1999) Feasibility and safety of ultrasound-aided thoracentesis in mechanically patients. Intensive Care Med 25:955-958
7. Lichtenstein D, Axler O (1993) Intensive use of general ultrasound in the intensive care unit. Prospective study of 150 consecutive patients. Intensive Care Med 19:353-355

8. Volpicelli G, Lamorte A, Tullio M, Cardinale L, Giraudo M, Stefanone V, Boero E, Nazerian P, Pozzi R, Frascisco MF (2013) Point-of-care multiorgan ultrasonography for the evaluation of undifferentiated hypotension in the emergency department. Intensive Care Med 39:1290-1298 